\title{
Resilience Factors as a Buffer against the Effects of Syndemic Conditions on HIV Risk and Infection among Tanzanian MSM
}

Adeniyi Adeboye ${ }^{1}$, Michael W Ross ${ }^{2}$, Michael J Wilkerson ${ }^{1}$, Andrew Springer ${ }^{1}$, Hycienth Ahaneku ${ }^{3}$, Rafeek A Yusuf ${ }^{3}$, Titilope 0 Olanipekun ${ }^{3}$ and Sheryl McCurdy ${ }^{3}$

${ }^{1}$ Department of Health Promotion and Behavioral Sciences, The University of Texas School of Public Health, Houston, TX, USA

${ }^{2}$ Program in Human Sexuality, Department of Family Medicine and Community Health, University of Minnesota, Minneapolis, MN, USA

${ }^{3}$ Department of Epidemiology, Human Genetics and Environmental Sciences, The University of Texas School of Public Health, Houston, TX, USA

"Corresponding author: Adeniyi Adeboye, Department of Health Promotion and Behavioral Sciences, The University of Texas School of Public Health, Houston, TX, USA, Tel: +447960266152; E-mail: adeboye05@yahoo.co.uk

Rec date: August 16, 2017; Acc date: September 25, 2017; Pub date: September 28, 2017

Copyright: ( 2017 Adeboye A, et al. This is an open-access article distributed under the terms of the Creative Commons Attribution License, which permits unrestricted use, distribution, and reproduction in any medium, provided the original author and source are credited.

\section{Abstract}

Background: In the extremely homophobic conditions in Tanzania and other countries in sub-Saharan Africa (SSA), men who have sex with men (MSM) experience disproportionate amounts of both HIV infection and psychosocial health problems, but no research has been conducted to examine the synergistic or additive effects of these adversities on their experiences. While the predominantly deficit-based research approach has identified various HIV risks and vulnerabilities, any naturally occurring protective and resilience factors that these men naturally employ to these health-related adversities remain undetermined.

Purpose: This study examined the syndemic effects of psychosocial health problems on HIV infection and HIV risk and simultaneously sought to identify any resilience or protective factors that may buffer against the syndemic production of HIV infection and risk behavior among high-risk Tanzanian MSM. These syndemic health problems were coined as SAVID (Substance abuse during condomless sex, childhood and adolescent sexual abuse, violence, internalized homonegativity and depression)

Methods: Cross-sectional data on demographic characteristics and HIV prevalence and risks among Tanzanian MSM residing in Dar es Salaam and Tanga were used to examine whether there is a proportional increase in HIV infection and risk as the number of syndemic conditions increases. Logistic regression analysis was used to test for interactions between syndemic conditions and to identify any potential resilience factors as a way of establishing protection against the syndemic production of HIV infection and risk among these men. At the level of interaction between protective resilience factors (moderators)-support, age visibility- and SAVID syndemic; those that reported high social visibility among Acquaintance have lower odds of contracting HIV infection when compared to those that have lower social visibility among acquaintances

Results: The results revealed significant additive associations between increasing numbers of SAVID syndemic components and higher rates of HIV infection and risk. Among a variety of identified potential resilience factors, three-functional socio-support, age at self-awareness of sexual orientation and formal social visibility among acquaintances-were found to directly associated with lower odds of contracting HIV infection. At the level of interaction between protective resilience factors (moderators: functional social support, age at awareness of sexual orientation, and formal social visibility among acquaintances) and SAVID syndemics, only those sampled MSM that reported high social visibility among acquaintances had lower odds of contracting HIV infection when compared to sampled MSM that had lower formal social visibility among acquaintances

Conclusion: Harnessing naturally-occurring resilience factors through strength-based research approach and innovatively disseminate them through existing secrete social network may be a cost effective and a novel health promotion strategy suitable for MSM in extreme homophobic environment.

Keywords: Sub-saharan africa; HIV infection; Antigay violence; Syndemics

\section{Introduction}

The health of men who have sex with men (MSM) is a global public health concern not only because this subpopulation is substantially affected by HIV epidemics but also because the healthcare needs of these men have been heightened by the syndemic production of this disease. Corollary to this are the behavioral HIV-intervention efforts, which have been characterized as being efficacious but insufficient as having low retention and as being too nonsustainable to reduce HIV transmission among these men. Hence, although current HIV interventions that target MSM are characterized as being suboptimally effective in some contexts, their notable absence in countries in subSaharan Africa (SSA) underscores the recent urgent call for another innovative approach to meeting the health needs of MSM in all settings [1-55]. 
Citation: Adeboye A, Ross MW, Wilkerson MJ, Springer A, Ahaneku H, et al. (2017) Resilience Factors as a Buffer against the Effects of Syndemic Conditions on HIV Risk and Infection among Tanzanian MSM. J Health Educ Res Dev 5: 230. doi: $10.4172 / 2380-5439.1000232$

Page 2 of 8

Further, current HIV interventions that target MSM parallel the predominant HIV epidemiologic behavioral-research approach, which is deficit based (i.e., risk identifying) rather than strength based, which could identify any natural resilience-based protective factors [18]. Previous empirical studies on MSM, with few exceptions, have focused only on problems, ignoring any strengths, resilience factors, and competencies that some of these men already employ naturally to successfully survive in extremely hostile environments. However, HIV interventions that parallel research grounded in strength-based approaches have been conceptualized as sustainable because they would promote naturally occurring protective factors, whereas those interventions informed by studies grounded in deficit-based approaches have been theorized to plausibly offer only time-limited effects [14].

In most countries in sub-Saharan Africa (SSA), where same-sex behaviors are criminalized, the research conducted to date (although admittedly deficit based) has documented useful information on the prevalence of HIV and health-related adversities among MSM in this region $[4,27,32,45]$. Nevertheless, the naturally protective strengths and resilience factors that MSM in SSA have been drawing on to successfully cope with these extreme health-related adversities are as yet undetermined.

\section{HIV disparities and syndemic conditions among MSM in SSA: Pathway to resilience}

Although studies focusing on behavioral resilience may be rare, and HIV interventions targeting MSM in SSA may also be scarce, numerous studies have documented a greater burden of HIV these men have to cope with in order to be resilient. For instance, while research advances have suggested that to achieve an AIDS-free generation, inclusion of MSM in HIV research interventions is imperative, the unmet health needs of MSM in this region are currently being heightened by discriminatory laws prohibiting these men from attaining a rights-defined health status $[3,48]$. Also, despite evidence suggesting that great strides have been made in HIV prevention among behaviorally heterosexual populations, the rates of HIV continue to increase disproportionately among MSM [55]. For example, in Kenya, the HIV prevalence among the general population ranged from $6.70 \%$ in 2003 to $5.60 \%$ in 2012 (Kenya AIDS response progress report 2014) while that of MSM was $24.60 \%$ during the same period [21,51]. Likewise, whereas the national prevalence of HIV in Zambia was documented at $15.20 \%$ among heterosexual adults, it was $33.30 \%$ among MSM [56-61]. A similar trend was documented among Tanzanian MSM in Zanzibar, with 12.3\% HIV prevalence while that of the local general population was $2.00 \%$ [9].

Beyond this HIV disparity, the psychosocial problems such as depression, internalized homonegativity (IH) (Ross and Anderson, 2014), childhood sexual abuse (CSA), antigay violence (Anderson et al.), and substance abuse were found to be prevalent and independently associated with HIV among MSM across countries in SSA $[2,9,36,38,45]$. Although syndemic theory has yet to be applied in the study of MSM in SSA, synthesis of the evidence has not only confirmed the existence of the HIV disparity but also strongly suggests the existence of a disproportionate level of multiple health problems that could plausibly affect the syndemic production of HIV among MSM.

\section{Resilience: A corollary of syndemic theory}

Although interest in syndemic applications in public health has grown in recent years, especially among MSM, the application of resilience theory as a way of mitigating or abating syndemic conditions among sexual minorities is still nascent in the United States and has never been quantitatively applied among MSM in SSA. Nevertheless, empiric applications of syndemic theory among MSM in the United States and and among marginalized women in South Africa have highlighted the coexisting and synergistic influence of multiple psychosocial health problems as drivers of HIV epidemics among these socially marginalized subpopulations $[30,39,54]$. The results of these studies offer a way of advancing HIV-prevention effectiveness among MSM in high-risk contexts, and some have been extended by resilience theory to identify protective factors that may mitigate the syndemic production of HIV among these sexual minorities [2,37].

Hence, in spite of the prevalence and impact of syndemic conditions on the health of MSM, synthesis of the evidence has shown that some MSM are resilient, especially in stopping tobacco use, overcoming internalized homonegativity $(\mathrm{IH})$ and avoiding drug use $[14,17,31,53]$. This suggests that not all MSM who reside in a hostile environment and are exposed to multiple psychosocial health problems and their syndemic effects develop negative health outcomes. For example, Stall et al. revealed that among MSM who had experienced three or more syndemic conditions, $22.00 \%$ developed HIV; conversely, however, this indicates that $78 \%$ of these men who are exposed to similar syndemic conditions are HIV negative [53]. Thus, HIV research harnessing resilience-related protective factors that these men employ to avert HIV may reveal a synergistic benefit on MSM in any high-risk context.

\section{Definition, evidence, and relevance of cultural resilience factors}

Resilience, defined and operationalized as positive adaptations despite exposure to health-related adversities has been applied in developmental psychology to positively advance the health of socially marginalized populations. It has also recently been posited in public health discourse as an effective resource and untapped HIV research framework toward broadening the scope of HIV interventions targeting MSM in high-risk contexts $[16,18,26]$. Those researchers critically evaluated HIV interventions that parallel deficit-based approaches in HIV research (i.e., risk identifying) and suggested HIV research grounded in strength-based approaches, instead, as an innovative way of advancing effective prevention science among MSM [18].

Recently, the existence of the resilience variables shamelessness, sexual creativity, social creativity, social activism, self-motoring, demographics (education), and social support have been identified by AIDS researchers in the domain of the health of sexual minority groups [18]. Among those posited behavioral resilience factors, shamelessness, optimism, demographics, and social support have been "unpacked" and operationalized empirically as risk modifiers. For instance, social support, optimism, and education were found to be effective in not only modifying the prevalence of HIV but also in positively affecting syndemic conditions among MSM in the United States $[5,23,37]$.

Further, although numerous studies documented the buffering effect of social support on health-related adversities among MSM emphasized the difference between structural and functional types of socio-support and the impact they have in counterbalancing stressful 
Citation: Adeboye A, Ross MW, Wilkerson MJ, Springer A, Ahaneku H, et al. (2017) Resilience Factors as a Buffer against the Effects of Syndemic Conditions on HIV Risk and Infection among Tanzanian MSM. J Health Educ Res Dev 5: 230. doi: $10.4172 / 2380-5439.1000232$

Page 3 of 8

life events $[2,8,59,60]$. This difference was confirmed when O'Leary et al. reported that functional- defined as social relationship that offers "tangible" assistance, but not structural socio-support- such as size of the social network confers a buffering effect on HIV risk behaviors among African American MSM [37].

In addition, social visibility, an extension of either direct or indirect disclosure of one's sexual orientation, especially to family members or friends, has been identified as a protective factor. For instance, in a qualitative study conducted among MSM in Beirut, Lebanon, Wagner et al. found that participants who had gained social visibility by disclosing their sexual orientation to family members, either directly or indirectly, tended to be more likely to use condoms consistently [58]. This finding supports the philosophy that underlies family-based HIV prevention programs, an innovative approach suggested by Garofalo et al. for use especially among young socially marginalized MSM. The concept of coping through stigma competence, as developed by Adams and Kimmel, empirically demonstrated the effectiveness of selfacceptance of one's homosexual identity as a protective factor against perceived stigma [10,13]. The literature provides similar evidence suggesting that managing homophobia at a younger age is an indication of early development of an adaptive coping technique that may later confer protection in an oppressive society [35,42]. Thus, those who are aware of their sexual orientation at a young age may have enough time to develop effective techniques for coping with HIVrelated adversities. For instance, gay-related developmental studies and resilience research have suggested and empirically confirmed that preadolescents' sense of their being different-i.e., their awareness of being gay-learned at early age may be a protective factor among gay people $[2,41,49,56,59]$. This is because by age 15 , adolescents are said to be cognitively capable of making good health-related decisions on the basis of their assessment of their own life situations [28]. On the other hand, although Coates et al. asserted that a delayed age of one's sexual debut could be a useful way to increase the effectiveness of HIV behavioral strategies for reducing HIV transmission among at-risk populations, it has yet to be explored as a protective factor among African MSM [7].

Nevertheless, owing to the varying effects of culturally based contextual factors, resilience factors that are deemed protective for MSM in less homophobic settings may not be protective in extremely homophobic settings. For example, as a result of societal homophobia, many MSM in SSA have adopted an outward heterosexual orientation through marriage and having sexual relationships with women. This subgroup of non-gay identified MSM in SSA has been described by many AIDS researchers as being a bridge for the transmission of HIV to the behaviorally heterosexual population [32,34]. This selfpreservational or protective behavior of these gender role-conforming MSM, in contrast with non-conforming MSM, could arguably be expected to occur in most traditional gender-sensitized SSA communities. Thus, in SSA, where gender-based ideas of homosexuality are prevalent, the assumption of a non-gay outward identity by MSM may be an adaptive strategy for protecting themselves against societal homophobic violence. This has been documented as a health-seeking strategy among South African and Tanzanian MSM $[24,29,48]$. Although the contentious concepts of "coming out" and "social visibility" have also been identified in other studies as protective factors leading to positive health outcomes in Western sample of MSM, they may be, however, counterproductive if adopted by MSM in homophobic cultural settings in SSA [44].

\section{Cultural resilience among MSM in SSA}

An extensive literature search revealed only one deliberate scientific inquiry that identified resilience factors among MSM in SSA, but inferential data in the extant literature reveal that a large number of MSM in SSA are resilient despite HIV-related adversities. In South Africa, Graziano et al. used a strength-based approach as a way of broadening the scope of HIV prevention by qualitatively applying a participatory-action research framework to identify both the adversities confronting MSM and the resilience-based protective factors they employ for coping with oppression [22]. In concurrence with the resiliency variables and constructs listed by Herrick et al. Graziano identified socio-support, optimism, a supportive socionetwork, empathy, a gay-affirmative religion, self-acceptance of being gay, and spirituality associated with religiosity as strengths and protective factors adopted by MSM in South Africa for coping with different forms of adversity related to their being homosexual [17]. On the other hand, Ross and Anderson et al. found religiosity to be associated with depression in Tanzanian MSM. McAllister et al. proposed a communication-activism strategy, termed "Tswanarisation," to promote creative interventions that support an already-existing culturally resilient and distinctive Setswana gay culture $[29,45]$. This process involves localizing and adapting behaviors of the Western gay culture into the existing Setswana gay culture as an innovative way of assuaging the homophobic experience of MSM in Botswana and other countries in SSA. It emphasizes sexual creativity, already identified as a strength and resilience factor by Herrick et al. as a strategy for safe sexual expression [16].

Tanzanian MSM, like those in any other resource-constrained and rights-challenged countries in SSA, are confronted by health-related adversities within the context of a pervasively homophobic environment. They are also socially marginalized, systematically excluded from HIV interventions and denied access to health services in a way that threatens the possibility of achieving an AIDS-free generation in Tanzania [48]. This poses a great challenge to HIV researchers and strongly indicates the urgent need for innovative HIVprevention approaches targeting not only Tanzanian MSM but all MSM in SSA. This may require identifying and empirically testing naturally occurring protective factors by applying strength-based research approaches that differ from the traditional deficit-based approaches to HIV research. However, SSA is culturally heterogeneous and it can't be assumed that the same factors will be consistent across different cultures.

\section{This Exploratory Study}

To add to the body of literature grounded in strength-based approaches, this study explored the potential moderating effect of both internal (e.g., delay in sexual debut) and external (e.g., socio-support) resources among Tanzanian MSM on the association between SAVID syndemic conditions - Substance abuse during condomless sexual intercourse, adolescent sexual abuse, violence, Internalized Homonegativity ( $\mathrm{IH})$ and Depression - and HIV risks and infection. In doing so, we aimed to address the following research questions:

1. Are there any correlational associations between SAVID Syndemic Components?

2. Are there any additive or synergistic associations between SAVID syndemic and HIV risk?

3. Are there any additive or synergistic associations between SAVID syndemic and HIV infection? 
Citation: Adeboye A, Ross MW, Wilkerson MJ, Springer A, Ahaneku H, et al. (2017) Resilience Factors as a Buffer against the Effects of Syndemic Conditions on HIV Risk and Infection among Tanzanian MSM. J Health Educ Res Dev 5: 230. doi: $10.4172 / 2380-5439.1000232$

Page 4 of 8

4. Do a combination of SAVID syndemic variables additively increase risk behaviors or HIV outcomes?

5. Do potential resilience factors such as, identifying as gay but still having a meaningful sexual relationship with a woman, the age at sexual debut, age at awareness of sexual orientation and sociovisibility (direct or indirect disclosure of sexual orientation) buffer (protect) the effects of syndemic conditions on HIV risk and infection?

By using the conceptual pathway (Figure 1) below proposed by Herrick et al.

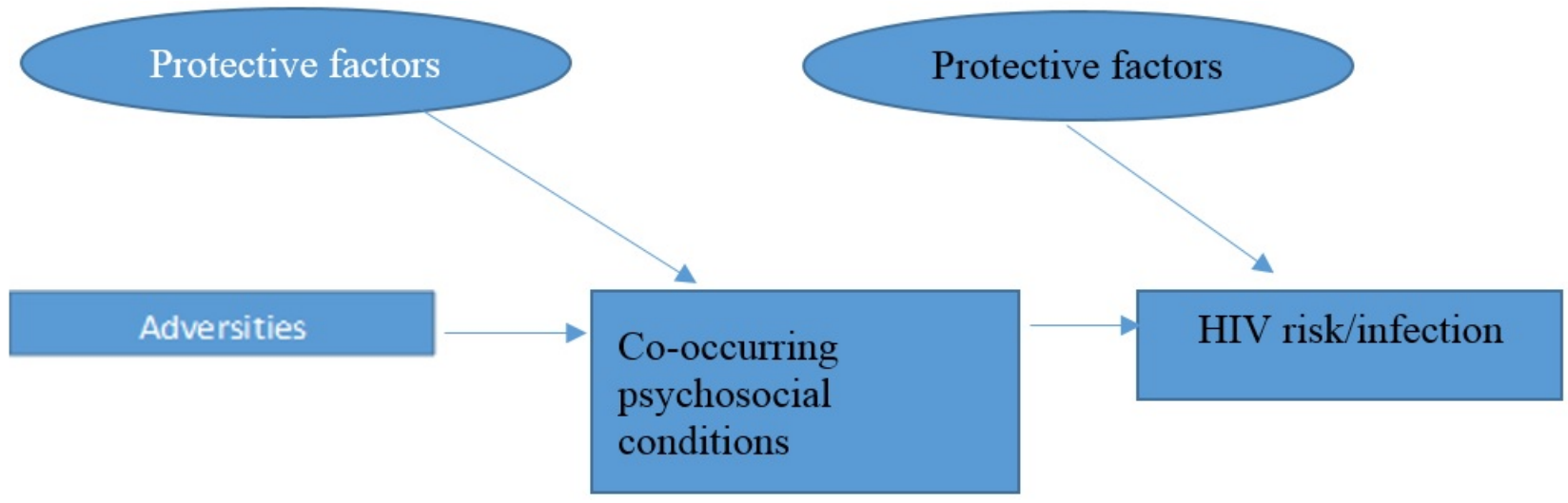

Figure 1: Conceptual pathway to identify protective factors in which protective factors may promote health and resilience, we hypothesized the following: 1. An increase in the number of coexisting syndemic conditions (Substance abuse during condomless sexual intercourse, adolescent sexual Abuse, Violence, Internalized homonegativity and Depression) is associated with increased levels of HIV risk behavior and higher revalence of HIV. 2. Potential resilience factors under study (level of social support, levels of socio-visibility, age at sexual debut, identifying as gay but still have a meaningful sexual relationship with women, age at awareness of sexual orientation) will directly confer protection against HIV risk and infection. 3. Moderating effects of potential resilience factors on SAVID syndemic components will significantly confer protection against HIV risks and infection

\section{Methods}

This study is based on a secondary analysis of data collected from a cross-sectional survey carried out in two cities in Tanzania during 2012 and 2013. The study population consisted of MSM residing in Dar e Salaam, a major city in Tanzania (estimated population, 3 million), and Tanga, a provincial city located between Dar es Salaam and Mombasa, Kenya. Two hundred MSM were recruited for the study from Dar es Salaam, and 100 were recruited from Tanga using the respondentdriven sampling method previously detailed by Ross et al. [45].

\section{Measures and data collection}

Data were collected using self-administered questionnaire comprising 11 sections: MSM network, terminology and life events, marriage and partnership, sexual behavior and condoms, sexual partner matrix, STI/HIV knowledge and risk perceptions, STI history, violence demographics and survey processing. Measures of $\mathrm{IH}$, depression were based on previously valid/reliable questions.

\section{Dependent variable}

The primary outcomes for this study were HIV seroprevalence and sexual risks:

1. HIV seroprevalence was determined by testing biologic samples provided by the subjects. Two rapid analytic test kits were used: for initial testing, the Determine for HIV 1/2 kit (Abbott Laboratories, Chicago, IL, USA), and for confirmation of the initial test, the Uni-Gold HIV kit (Trinity Biotech PLC, Bray, County Wicklow, Ireland). If the results were discordant, that of the Uni-Gold test was used. Validation studies previously conducted in five African countries showed that the Abbott Determine kit has a sensitivity of $99.80 \%$ and a specificity of $99.40 \%$, and the Trinity Uni-Gold kit has a sensitivity of $98.50 \%$ and a specificity of $99.50 \%$. These tests require minimal infrastructure and provide results quickly [40].

2. The second outcome was constructed as the risk variable "ever had condomless sexual intercourse," which took into account the number of times of having condomless sexual intercourse including vaginal and receptive and insertive anal sex with casual and/or regular partners and/or commercial sex workers. This outcome is a dichotomous variable determined by the participants' self-reported condomless sexual intercourse (yes or no) during the 6 months immediately prior to the survey.

\section{Independent variables}

Demographics: The sociodemographic characteristics examined were age, employment status, level of income, and level of education completed.

Syndemic factors: Each of the following syndemic factors analyzed was previously identified as a contributor to a syndemic effect on sexual HIV risk and/or seroprevalence.

1. Substance use during sex: To quantify this variable, the responses to the following two-part question were considered: "How many of those times that you received anal sex/had insertive anal sex with all partners and did not use a male condom were you high or drunk?" The summed response to these two questions, which 
Citation: Adeboye A, Ross MW, Wilkerson MJ, Springer A, Ahaneku H, et al. (2017) Resilience Factors as a Buffer against the Effects of Syndemic Conditions on HIV Risk and Infection among Tanzanian MSM. J Health Educ Res Dev 5: 230. doi: $10.4172 / 2380-5439.1000232$

Page 5 of 8

were developed specifically for this study, was used as the variable.

2. Childhood sexual abuse (CSA): This was defined according to the developmental classification of Doll et al. [11]. According to that schema (Appendix B), an experience is classified as abusive contingent on the age of the child or adolescent involved, the difference in age between the partners (i.e., peer or non-peer contact), whether anal penetration occurred, and whether force was involved. Any contact involving force was considered sexual abuse regardless of age. Participants were asked, "Have you ever been forced or coerced to have sex with someone?" "How old were you when you were forced or coerced into having sexual contact with someone?" "How old was the person who first forced you or coerced you into having sexual contact?" "Was your first sex partner a man or woman?" "How old was your first sex partner?" If the responses to any of these questions implied CSA, the participant was considered to have experienced or to have a history of such abuse.

3. Level of antigay violence experienced: Four types of abuse were considered. Participants could answer "Yes", "No" or "No response" to the four-part question, "Have you been the victim of physical violence or abuse, verbal abuse, moral abuse, or sexual abuse?" Each part of the question was asked separately, so the maximum possible score was 4 . The variable was dichotomized according to the number of "Yes" answers: the level of violence was characterized as "high" when the score was 3 or 4 and as "low" when the score was 2 or less. These items used to establish the level of antigay violence were created specifically for this study. Cronbach's Alpha score for this sample was 0.714

4. Stigma, as indicated by the IH score: $\mathrm{IH}$ is the acceptance and internalization of negative attitudes toward one's own sexual orientation [50]. It was measured by using an 8-item short version of the 28-item "Reactions to Homosexuality Scale" $[46,52]$. This short version, which uses a 6-point Likert scale from 0 (strongly disagree) to 5 (strongly agree), is reliable and valid in MSM populations in SSA [47]. An individual's score ranges from 0 to 40.

5. Depression: This factor was identified by using the Patient Health Questionnaire-9 (Appendix A, PHQ-9), which is a validated instrument for measuring the nine diagnostic criteria of depressive disorders listed in the Diagnostic and Statistical Manual of Mental Disorders, fourth edition (DSM-IV). The PHQ-9 is reliable and valid for both making a criterion-based diagnosis and measuring the severity of depression [22]. The instrument asks the subject to score each of the nine DSM-IV depressive symptoms from 0 (not present at all) to 3 (present nearly every day); thus, the score ranges between 0 and the PHQ-9 has been used in sub-Saharan populations and has been shown to be reliable and valid $[1,33]$. Cronbach's Alpha score for this sample was 0.867

6. The SAVID Syndemic: This is the acronym formed by the five psychosocial factors just listed. In this study, grouping of the MSM followed the pattern employed by Stall et al. Thus, the variable to describe the SAVID syndemic was created by categorizing subjects into four mutually exclusive groups according to their experience during the 6 months preceding the survey. Those groups were defined as (1) no reported experiences of any of the psychosocial factors (i.e., substance use during sex, history of CSA, experience of antigay violence, IH, and depression), (2) one SAVID syndemic factor reported, (3) two of the factors reported, and (4) three or more of the factors reported.

\section{Resilience factors}

Social support: By considering the possible varying effects of structural and functional measures of social support as stated by Cohen and Wills (1985), two variables were constructed to assess the effects of social support on HIV seroprevalence and risks.

Structural socio-support: This variable takes into account the size of the participants' social network according to their responses to the question, "How many gay people do you know personally by name (and who know you as well) who are living in this city, whom you have seen in the last month, and who are at least 15 years old. The answer to this question, based on the normal distribution of the responses, was dichotomized as High or Low structural socio-support using mean score as cutoff number

Functional socio-support: This was determined by the question, "How many gay or bisexual men do you consider close friends; i.e., men you can rely on for help and you, would help if needed?" Participants' answers were dichotomized as High or Low functional socio-support using average score.

Age at sexual debut: This variable was determined according to each participant's answer to the question, "How old were you when you had sex for the first time with anyone?" Empirical evidence underpins the assertion of Coates et al. that delaying the age at which the person had his first sexual intercourse would be effective in reducing HIV transmission [7]. In accordance with that evidence, the participants' answers were first averaged, and then each man's answer was dichotomized as below or above that average age; an above-average age at sexual debut was treated as indicating a protective factor.

Household income: Three levels of household income were used: I don't know $=0$, less than $150,000 \mathrm{TSH}=1$, and at least $150,000 \mathrm{TSH}=2$ $(10,000 \mathrm{TSH}=\$ \mathrm{US} 4.50)$. Participants could also choose to provide no response.

Socio-visibility: Direct or indirect disclosure of sexual orientation to friends, family, and/or coworkers was determined by the participants' answers to the following questions: Do any of your female/male friends know you have sex with men? Does anyone in your immediate family (i.e., wife, mother, father, brother, sister) know you have sex with men? Do any of your coworkers, employers, nurses, or doctors know you have sex with men (i.e., formal socio-visibility)? Each participant's answers were dichotomized as Low or High socio-visibility using the average score as cutoff number

Educational level: This was dichotomized as primary or postprimary educational attainment.

Identifying as gay but still having a meaningful sexual relationship with women: To determine this variable, four categories of sexual orientation were taken into consideration. Participants could selfidentify as "gay," "straight," or "bisexual" in answer to the direct question, "Do you consider yourself to be gay/homosexual, straight, or bisexual?" Those who answered "gay" and also answered "Yes" to the follow-up question, "Do you have any meaningful sexual relationships with women?" were categorized as "identifying as gay but still having a meaningful sexual relationship with women."

Age at awareness of sexual orientation: This age was determined by the answer to the question, "How old were you when you first became aware that you were sexually attracted to other men?" Participants' 
Citation: Adeboye A, Ross MW, Wilkerson MJ, Springer A, Ahaneku H, et al. (2017) Resilience Factors as a Buffer against the Effects of Syndemic Conditions on HIV Risk and Infection among Tanzanian MSM. J Health Educ Res Dev 5: 230. doi: $10.4172 / 2380-5439.1000232$

Page 6 of 8

answers were averaged, and each man's answer was dichotomized according to the mean age. This helped to confirm the phenomenon described by Morrow (2001) that those men who become aware of their sexual orientation at a relatively younger than older age have more time to develop adaptive coping techniques; in this context, toward HIV-related adversities.

\section{Data Analysis}

To establish whether the psychosocial health problems under study overlap to form SAVID syndemic variable, Pearson product-moment correlation testing was performed. Chi-squared tests of association was also carried out to determine the levels and statistical significance of the HIV-risk behavior and infection outcomes for the different numbers of SAVID syndemic factors reported $(0,1,2$, or 3 or more).

To identify the potential buffering effects, the statistical significance of the interactions between the SAVID syndemic variable and each of the potential resilience factors was determined for the two outcomes in logistic regression models. Statistical significance testing was two tailed, and $\mathrm{p}$-value was set at $<0.05$. All analyses were carried out using SPSS version 22 software.

\section{Results}

The demographic and other characteristics of the 300 Tanzanian MSM are denoted Slightly more than half $(51.30 \%)$ of the participants were younger than 23 years old, signifying that the sample population was young. Sixty-two percent of the men had attained a post-primary school education. The rate of unemployment was high, $81 \%$. About one third of the sample tested positive to HIV, of which $92.00 \%$ were new infections in men who believed that they were HIV negative, and about three quarters reported "ever had condomless sexual intercourse" with either a man or a woman in the 6 months immediately preceding the time the data were collected.

It presents the prevalence of the SAVID syndemic and potential resilience factors. A third of the sample population reported a history of substance use while having unprotected sexual intercourse. One third of the sample also reported having a history of CSA. More than one in $5(22.00 \%)$ reported having experienced violence associated with homophobia. More than 1 reported having a high level of $\mathrm{IH}$, and almost half had a high level of depression.

The intercorrelations among the psychosocial health problems examined. The significant overlap in correlation reflects the syndemic theory, which posits the existence of clustering relationships among the components of the syndemic factor.

The percentage of men reporting high-risk sexual behavior and HIV infection increases as the number of syndemic conditions experienced increases, confirming the results of previous studies.

Finally, the results of the analyses of interactions between the syndemic burden and the potential resilience factors. Two of the factors-functional socio-support, and age at awareness of sexual orientation - had an inverse association with HIV seroprevalence. In the direct model, they both reduce the odd of contracting HIV infection. As moderators, functional social support and age at awareness of sexual orientation were also found to reduce the odd of contracting.

\section{Discussion}

To the best of our knowledge, this is the first study to be conducted in SSA among MSM that supports the view that using a strength-based approach to HIV research can identify resilience factors that may buffer the effects of syndemic conditions on HIV-risk behavior and infection. Employment status, educational level, identifying as gay but still having a meaningful sexual relationship with women, level of social visibility, functional and structural socio-support, age at awareness of sexual orientation, and age at sexual debut were among the identified potential resilience factors tested for their buffering effects. None of these potential resilience factors on their own demonstrated a buffering effect against the syndemic production of HIV-risk behavior, but three factors proved to be buffers against the syndemic production of HIV infection: age at awareness of sexual orientation, functional socio-support and formal social visibility among acquaintances. The results revealed significant additive associations between increasing numbers of SAVID syndemic components and higher rates of HIV infection and risk. Functional socio-support, age at self-awareness of sexual orientation and formal social visibility among acquaintances-were found to be directly associated with lower odds of contracting HIV infection. At the level of interaction between protective resilience factors (moderators) - that is functional social support, age at awareness of sexual orientation, and formal social visibility among acquaintances-and SAVID syndemic, only those MSM sampled who reported high social visibility among acquaintances had lower odds of contracting HIV infection when compared to sampled MSM that had lower formal social visibility among acquaintances

These results extend those of previous studies grounded in resilience theory carried out among MSM in the United States, showing that Tanzanian MSM also possess resilience factors that buffer the effects of multiple psychosocial health problems on HIV infection [2,23,37]. For instance, earlier studies on the moderating effects of sense of competency manifested in locus of control on stress defined young MSM as either "internals" or "externals", with the latter demonstrating higher levels of self-esteem and reporting fewer psychological and physical symptoms $[2,25,28,59]$. This may be the reason why we found a buffering effect against HIV infection among the Tanzanian MSM who reported earlier age of sexual awareness of sexual orientation despite their constant exposure to societal homophobia. Our results also confirmed the work of Cohen and Wills et al. and replicated one of the findings of O'Leary et al. by revealing that functional support (i.e., actual support) rather than structural support (i.e., a measure of social network) buffers against the syndemic production of HIV infection $[8,37]$. Future research is needed, however, to explore the mechanisms underlying the interplay between these protective factors that provide a buffering effect on the syndemic production of HIV risk and infection.

Studies have revealed that social-visibility ("coming out") has been associated with equivocal health outcomes $[43,58]$. In other words, "coming out" could offer protection against or predispose to HIV risk and infection. Alternatively, sufficient self-confidence to come out might also be the determining factor. However, in this study, results showed that high social visibility among acquaintances (direct or indirect disclosure of sexual orientation) is associated with lower odds of contracting HIV infection and significantly and positively moderate the odd of syndemic production of HIV infection.

Several limitations need to be considered while interpreting the findings from this study. Although respondent-driven sampling, which is a nonbiased method, was used to recruit participants for the study, it 
Citation: Adeboye A, Ross MW, Wilkerson MJ, Springer A, Ahaneku H, et al. (2017) Resilience Factors as a Buffer against the Effects of Syndemic Conditions on HIV Risk and Infection among Tanzanian MSM. J Health Educ Res Dev 5: 230. doi: $10.4172 / 2380-5439.1000232$

Page 7 of 8

is nonprobabilistic and thus makes our findings less generalizable to other subpopulations. Some of the potential resilience factors we measured and analyzed (age at sexual debut and age at awareness of being gay) might also have introduced some bias because the participants provided this information retrospectively. Men who agreed to take part in the study were likely to be more connected with gay networks and more out, providing limited variance on these variables. Finally, the cross-sectional design of this study limits the findings to only a correlational level and does not allow cause-andeffect attribution.

\section{Conclusion}

Questions among prevention scientists about how to package and deliver effective HIV interventions to MSM in extremely homophobic environments, such as in Tanzania, may be answered through the conduct of innovative, strength-based studies. For instance, Coates et al. suggested that a network-based intervention may be an innovative approach for use among groups of stigmatized and vulnerable people, like MSM, who are systematically alienated from health services [7]. This may involve gaining access to the "secret" sexual network by identifying key individuals and training them for the purpose of disseminating HIV-protective strategies.

As Luthar et al. indicated, if research is fundamentally applied in the natural setting, it would be logical to focus on resilience-related protective factors that have the potential to positively affect at-risk individuals [26]. Because it is increasingly being recognized that MSM in SSA are not only disproportionately affected by HIV but are also exposed to syndemic-related adverse conditions, they are therefore a subpopulation well suited to the application of resilience theory. Thus, by identifying resilience factors that have buffering effects on syndemic conditions existing among Tanzanian MSM, HIV interventions for these men may one day become real.

The institutional review boards at The University of Texas Health Science Center (HSC-SPH-10-0033) and the Tanzanian National Institute for Medical Research (NIMR/HQ/R.8a/Vol.IX/1088) reviewed and approved the protocol for this study.

\section{References}

1. Adewuya AO, Ola BA, Afolabi OO (2006) Validity of the patient health questionnaire (PHQ-9) as a screening tool for depression amongst nigerian university students. Journal of Affective Disorders 96: 89-93.

2. Anderson AL (1998) Strengths of gay male youth: An untold story. Child and Adolescent Social Work Journal 15: 55-71.

3. Beyrer C, Sullivan PS, Sanchez J, Dowdy D, Altman D, et al. (2012) A call to action for comprehensive HIV services for men who have sex with men. The Lancet 380: 424-438.

4. Bui TC, Nyoni JE, Ross MW, Mbwambo J, Markham CM, et al. (2014) Sexual motivation, sexual transactions and sexual risk behaviors in men who have sex with men in Dar es Salaam, Tanzania. AIDS and Behavior 18: 2432-2441.

5. Buttram ME, Kurtz SP, Surratt HL (2013) Substance use and sexual risk mediated by social support among black men. Journal of Community Health 38: 62-69.

6. Chelugel B, Baltazar G, Orege P, Ibrahim M, Marum L, et al. (2006) Evidence for population level declines in adult HIV prevalence in kenya. Sexually Transmitted Infections 82: i21-i26.

7. Coates TJ, Richter L, Caceres C (2008) Behavioural strategies to reduce HIV transmission: How to make them work better. The Lancet 372: 669-684.
8. Cohen S, Wills TA (1985) Stress, social support, and the buffering hypothesis. Psychological Bulletin 98: 310.

9. Dahoma M, Johnston LG, Holman A, Miller LA, Mussa M, et al. (2011). HIV and related risk behavior among men who have sex with men in zanzibar, tanzania: Results of a behavioral surveillance survey. AIDS and Behavior 15: 186-192.

10. David S, Knight BG (2008) Stress and coping among gay men: Age and ethnic differences. Psychology and Aging 23: 62.

11. Doll LS, Joy D, Bartholow BN, Harrison JS, Bolan G, et al. (1992) Selfreported childhood and adolescent sexual abuse among adult homosexual and bisexual men. Child Abuse \& Neglect 16: 855-864.

12. Fenton KA (2010) Prevention with HIV-positive men who have sex with men: Regaining lost ground. Sexually Transmitted Infections 86: 2-4.

13. Garofalo R, Mustanski B, Donenberg G (2008) Parents know and parents matter; is it time to develop family-based HIV prevention programs for young men who have sex with men? Journal of Adolescent Health 43: 201-204.

14. Greenwood GL, Paul JP, Pollack LM, Binson D, Catania JA, et al. (2005) Tobacco use and cessation among a household-based sample of US urban men who have sex with men. American Journal of Public Health 95: 145-151.

15. Guzman R, Colfax GN, Wheeler S, Mansergh G, Marks G, et al. (2005) Negotiated safety relationships and sexual behavior among a diverse sample of HIV-negative men who have sex with men. Journal of Acquired Immune Deficiency Syndromes 38: 82-86.

16. Herrick AL, Lim SH, Wei C, Smith H, Guadamuz T, et al. (2011) Resilience as an untapped resource in behavioral intervention design for gay men. AIDS and Behavior 15: 25-29.

17. Herrick AL, Stall R, Chmiel JS, Guadamuz TE, Penniman T, et al. (2013) It gets better: Resolution of internalized homophobia over time and associations with positive health outcomes among MSM. AIDS and Behavior 17: 1423-1430.

18. Herrick AL, Stall R, Goldhammer H, Egan JE, Mayer KH (2014) Resilience as a research framework and as a cornerstone of prevention research for gay and bisexual men: Theory and evidence. AIDS and Behavior 18: 1-9.

19. Johnson WD, Diaz RM, Flanders WD, Goodman M, Hill AN, et al. (2008) Behavioral interventions to reduce risk for sexual transmission of HIV among men who have sex with men. The Cochrane Library.

20. Johnston LG, Holman A, Dahoma M, Miller LA, Kim E, et al. (2010) HIV risk and the overlap of injecting drug use and high-risk sexual behaviours among men who have sex with men in zanzibar (unguja), tanzania. International Journal of Drug Policy 21: 485-492.

21. Kenya AIDS response progress report 2014. Progress towards zero.

22. Kroenke K, Spitzer RL, Williams JB (2001) The Phq-9. Journal of General Internal Medicine 16: 606-613.

23. Kurtz SP, Buttram ME, Surratt HL, Stall RD (2012) Resilience, syndemic factors, and serosorting behaviors among HIV-positive and HIV-negative substance-using MSM. AIDS Education and Prevention: Official Publication of the International Society for AIDS Education 24: 193-205.

24. Lane T, Mogale T, Struthers H, McIntyre J, Kegeles SM (2008) "They see you as a different thing": The experiences of men who have sex with men with healthcare workers in south african township communities. Sexually Transmitted Infections 84: 430-433.

25. Lefcourt HM, Martin RA, Saleh WE (1984) Locus of control and social support: Interactive moderators of stress. Journal of Personality and Social Psychology 47: 378.

26. Luthar SS (2006) Resilience in development: A synthesis of research across five decades.

27. Magesa DJ, Mtui LJ, Abdul M, Kayange A, Chiduo R, et al. (2014) Barriers to men who have sex with men attending HIV related health services in dar es salaam, tanzania. Tanzania Journal of Health Research 16.

28. Mann L, Harmoni R, Power C (1989) Adolescent decision-making: The development of competence. Journal of Adolescence 12: 265-278. 
Citation: Adeboye A, Ross MW, Wilkerson MJ, Springer A, Ahaneku H, et al. (2017) Resilience Factors as a Buffer against the Effects of Syndemic Conditions on HIV Risk and Infection among Tanzanian MSM. J Health Educ Res Dev 5: 230. doi: $10.4172 / 2380-5439.1000232$

Page 8 of 8

29. McAllister J (2013) Tswanarising global gayness: The 'unAfricanargument, western gay media imagery, local responses and gay culture in botswana. Culture, Health \& Sexuality 15: 88-101.

30. McCarthy K, Wimonsate W, Guadamuz T, Varangrat A, Thienkrua W, et al. (2010) Syndemic analysis of co-occurring psychosocial health conditions and HIV infection in a cohort of men who have sex with men (MSM) in bangkok, Thailand. International AIDS Conference 18-23.

31. Mills TC, Paul J, Stall R, Pollack L, Canchola J, et al. (2004) Distress and depression in men who have sex with men: The urban men's health study. American Journal of Psychiatry 161: 278-285.

32. Mmbaga E, Dodo M, Leyna G, Moen K, Leshabari M (2012) Sexual practices and perceived susceptibility to HIV infection among men who have sex with men in dar es salaam. Mainland Tanzania. J AIDS Clinic Res 1: 12 .

33. Monahan PO, Shacham E, Reece M, Kroenke K, Ong'or WO, et al. (2009) Validity/reliability of PHQ-9 and PHQ-2 depression scales among adults living with HIV/AIDS in western kenya. Journal of General Internal Medicine 24: 189-197.

34. Morris M, Kretzschmar M (1997) Concurrent partnerships and the spread of HIV. AIDS 11: 641-648.

35. Morrow DF (2001) Older gays and lesbians: Surviving a generation of hate and violence. Journal of Gay \& Lesbian Social Services 13: 151-169.

36. Needle R, Kroeger K, Belani H, Achrekar A, Parry CD et al. (2008). Sex, drugs, and HIV: Rapid assessment of HIV risk behaviors among streetbased drug using sex workers in durban, south africa. Social Science \& Medicine 67: 1447-1455.

37. O'Leary A, Jemmott III JB, Stevens R, Rutledge SE, Icard LD (2014) Optimism and education buffer the effects of syndemic conditions on HIV status among african american men who have sex with men. AIDS and Behavior 18: 2080-2088.

38. Pereda N, Guilera G, Forns M, Gómez-Benito J (2009) The prevalence of child sexual abuse in community and student samples: A meta-analysis. Clinical Psychology Review 29: 328-338.

39. Pitpitan EV, Kalichman SC, Eaton LA, Cain D, Sikkema KJ, et al. (2013) Co-occurring psychosocial problems and HIV risk among women attending drinking venues in a south african township: A syndemic approach. Annals of Behavioral Medicine 45: 153-162.

40. Piwowar-Manning EM, Tustin NB, Sikateyo P, Kamwendo D, Chipungu C, et al. (2010) Validation of rapid HIV antibody tests in 5 african countries. Journal of the International Association of Physicians in AIDS Care 9: 170-172.

41. Plummer K (1975) Sexual stigma: An interactionist account Routledge and Kegan Paul London.

42. Quam JK, Whitford GS (1992) Adaptation and age-related expectations of older gay and lesbian adults. The Gerontologist 32: 367-374.

43. Rosario M, Hunter J, Maguen S, Gwadz M, Smith R (2001) The comingout process and its adaptational and health-related associations among gay, lesbian, and bisexual youths: Stipulation and exploration of a model. American journal of community psychology 29: 133-160.

44. Rosario M, Schrimshaw EW, Hunter J (2004) Ethnic/racial differences in the coming-out process of lesbian, gay, and bisexual youths: A comparison of sexual identity development over time. Cultural Diversity and Ethnic Minority Psychology 10: 215.

45. Ross MW, Anderson AM (2014) Relationships between importance of religious belief, response to anti-gay violence, and mental health in men who have sex with men in east africa. Periodical 25: 160.
46. Ross MW, Rosser B (1996) Measurement and correlates of internalized homophobia: A factor analytic study. Journal of Clinical Psychology 52: 15-21.

47. Ross MW, Kajubi P, Mandel JS, McFarland W, Raymond HF (2013) Internalized homonegativity/homophobia is associated with HIV-risk behaviours among ugandan gay and bisexual men. International Journal of STD \& AIDS 24: 409-413.

48. Ross MW, Nyoni J, Larsson M, Mbwambo J, Agardh A, et al. (2015) Health care in a homophobic climate: The SPEND model for providing sexual health services to men who have sex with men where their health and human rights are compromised. Global Health Action 8: 26096.

49. Savin-Williams RC (1995) Lesbian, gay male, and bisexual adolescents. Lesbian, Gay, and Bisexual Identities Over the Lifespan: Psychological Perspectives 165-189.

50. Shidlo A (1994) Internalized homophobia: Conceptual and empirical issues in measurement. Some of the Information in this Chapter was Presented at the Meeting of the American Psychological Assn, New York.

51. Smith AD, Tapsoba P, Peshu N, Sanders EJ, Jaffe HW (2009) Men who have sex with men and HIV/AIDS in sub-saharan africa. The Lancet 374: 416-422.

52. Smolenski DJ, Diamond PM, Ross MW, Rosser BS (2010) Revision, criterion validity, and multigroup assessment of the reactions to homosexuality scale. Journal of Personality Assessment 92: 568-576.

53. Stall R, Paul JP, Greenwood G, Pollack LM, Bein E, et al. (2001) Alcohol use, drug use and alcohol-related problems among men who have sex with men: The urban men's health study. Addiction 96: 1589-1601.

54. Stall R, Mills TC, Williamson J, Hart T, Greenwood G, et al. (2003) Association of co-occurring psychosocial health problems and increased vulnerability to HIV/AIDS among urban men who have sex with men. American Journal of Public Health 93: 939-942.

55. Sullivan PS, Carballo-Diéguez A, Coates T, Goodreau SM, McGowan I, et al. (2012) Successes and challenges of HIV prevention in men who have sex with men. The Lancet 380: 388-399.

56. Troiden RR (1988) Homosexual identity development. Journal of Adolescent Health Care 9: 105-113.

57. Truong HM, Kellogg T, Klausner JD, Katz MH, Dilley J, et al. (2006) Increases in sexually transmitted infections and sexual risk behaviour without a concurrent increase in HIV incidence among men who have sex with men in san francisco: A suggestion of HIV serosorting? Sexually Transmitted Infections 82: 461-466.

58. Wagner GJ, Aunon FM, Kaplan RL, Rana Y, Khouri D, et al. (2012) A qualitative exploration of sexual risk and HIV testing behaviors among men who have sex with men in beirut, lebanon. PloS ONE 7: e45566.

59. Walker LS, Greene JW (1987) Negative life events, psychosocial resources, and psychophysiological symptoms in adolescents. Journal of Clinical Child Psychology 16: 29-36.

60. Williams T, Connolly J, Pepler D, Craig W (2005) Peer victimization, social support, and psychosocial adjustment of sexual minority adolescents. Journal of Youth and Adolescence 34: 471-482.

61. Zulu K, Bulawo N, Zulu W (2006) Understanding HIV risk behaviour among men who have sex with men in zambia. AIDS 13-18. 